\title{
Adjustment of p-Value Expression To Ontology Using Machine Learning For Genetic Prediction, Prioritization, Interaction, And Its Validation In Glomerular Disease
}

Boutaina Ettetuani ( $\square$ ettetuani.boutaina@gmail.com )

Systems and Data Engineering Team

Rajaa Chahboune

Abdelmalek Essaadi University

Ahmed Moussa

Systems and Data Engineering Team

\section{Research Article}

Keywords: Glomerular diseases, Gene expression, Gene ontology, Machine learning, omics sciences, Gene prediction, Gene prioritization, Gene interaction

Posted Date: February 14th, 2022

DOI: https://doi.org/10.21203/rs.3.rs-1269037/v1

License: (c) (1) This work is licensed under a Creative Commons Attribution 4.0 International License.

Read Full License 


\title{
RESEARCH
}

\section{Adjustment of p-value expression to ontology using machine learning for genetic prediction, prioritization, interaction, and its validation in glomerular disease}

\author{
Boutaina ETTETUANI ${ }^{*}$, Rajaa CHAHBOUNE ${ }^{2}$ and Ahmed MOUSSA ${ }^{1}$
}

\footnotetext{
${ }^{*}$ Correspondence:

ettetuani.boutaina@gmail.com

${ }^{1}$ Systems and Data Engineering

Team, National School of Applied

Sciences, Abdelmalek Essaadi

University, Tangier, MOROCCO

Full list of author information is

available at the end of the article
}

\begin{abstract}
Background: The selection of gene expression mixed to multiple similarity scores of their gene ontology terms (GO), were adjusted by our algorithm as essential prediction tasks for evaluating the regulatory pathways. Moreover applying machine learning techniques, in order to link the gene products into networks that prioritize candidate genes as classification. Finally detecting some significant genetic interactions by applying our algorithm model as validation of the results.
\end{abstract}

Results: Experimental validation of all associations facilitates the discovery of causative genes known to be related to glomerular diseases (GD). As well as EGR1, IL33, BMP2, SLAMF8. In which their GO annotations related to this gene include kidney vasculature development, regulation of cell activation/ inflammatory/immune effector/adaptive immune/glomerulus/glomerular mesangial cell proliferation development, etc. Other genes as TNXA, FCER1A, NME3, FMOD, BTG2, PTGER4, AXL, CYP1A2, CYTL1, BHLHE40, IFI16, SPON1, ETNPPL, COL14A1, ITGAV, MYOZ2, CAMK2A, SORT1, RANBP1, in which their variants information include complement a set of $\mathrm{C} 3, \mathrm{C} 4$, and $\mathrm{C} 7$ measurement, serum $\operatorname{lgE} / \lg \mathrm{A}$ measurement, c-reactive protein measurement, nephrotic syndrome, immune system disease, tuberculosis, glomerular filtration rate, chronic kidney disease, etc. The lasts enable a rapid interpretation of complex gene expression studies. As well, other high-throughput genomics assays illustrate an overview of a computational model for gene prioritization and their genetic interactions.

Conclusions: The furtherance of combining and adjusting the genes expression to multiple similarity scores of their gene ontology (GO) and interacting all possible cross-association of the results reported classification of genes between among to the subject category and validated the abstracts.

Keywords: Glomerular diseases; Gene expression; Gene ontology; Machine learning; omics sciences; Gene prediction; Gene prioritization; Gene interaction

\section{Background}

The term C3 glomerulopathy (C3G) is a group of related conditions that cause the malfunction of the kidneys [1], characterized by the presence of glomerular deposits composed of C3 [2]. These glomerular diseases (GD) include many conditions with a variety of genetic/environmental causes [3].C3G is associated with changes (mutation) in many genes. Most of these genes provide instructions for making 
proteins that help to regulate a part of the body's immune response known as the complement system [4]. This system works together as a group of proteins to destroy foreign invaders/trigger/inflammation. A specific mutation in the complement system-related genes, like C3, ADAM19, ADAMTS13, C3AR1, C8A, CD46, CFB, CFD, CFI, CFHR(1-5) and as well as other complement system-related genes $[5,6]$, risk haplotypes of $\mathrm{CFH}$ and $\mathrm{CD} 46$ have been identified that modify disease penetration and severity $[7,8]$. In most cases, the cause of the $\mathrm{C} 3 \mathrm{G}$ is unknown.

Many kinds of research are still devoted to discovering new gene selection techniques. Likewise, the confidence of using a single criterion for genes selection is not always adopted, considering which criterion should be good. This question has inspired us with the idea of taking into account the ranking of evaluating p-value expression and proposing a novel gene selection for the transcriptomics data, focusing on the adjustment of gene expression to the similarity score. Furthermore, our solution can be considered the most informative and stable method for gene prediction/selection. Also, the gene expression results consist of the input of normalized gene expression measurements [9]. From this, a linear model fit for all genes is computed to find genes with significant differential expression (DE) between different conditions from understudied raw data (as a set of binary files in CEL format), accessible via the public repository of microarray data NCBI Gene Expression Omnibus (GEO) [10]. Moreover, the first step in the pre-processing is the quality control of the data because this latter is an essential step in any analytical process as a relative concept that depends on the nature of the biological sample, the experimental settings, and other factors. The full Robust Multi-array Average (RMA) was also performed to background-correct, normalize and summarize the process $[11,12]$. The organization of this article consists of two large sections. The first one (methods) includes gene signature identification of Differential Expressed (DE) genes, then a followed step leading to construct the workflow and their structure, prioritizing and interacting gene products on each cluster. Finally, a result section covers a set of interpretations of the biological/clinical results of our hypothesis.

\section{Methods}

A major focus of this follow-up study was demonstrated in (Fig. 1), proposing and validating a novel matrix-Expression-Similarity-Frequency; consisting of a new scoring scheme; based on a given combined/adjusted linear DE measurement selection in diverse experimental conditions of individual samples. Machine learning tasks $[13,14,15]$ then were used focusing on combining mathematical algorithms to our gene products by clustering this last as unsupervised learning first based on their normalized matrix-Expression-Similarity-Frequency scores of occurrence to find out the structure as groups of a similar category. The data, in this case, contains only inputs and no desired output labels. Then a classification as the second step where the algorithm contains both the inputs and the desired outputs (limited to a set of outputs), used to construct co-regulation, and link them into networks that prioritize candidate genes as a logistic regression tool. In addition, significant genetic interactions were detected for a specific tissue type, genetic background, experimental stimulus, or clinical variables as validation of the results. 


\section{Experimental Data}

Five datasets (Table. 1) consisting of human kidney biopsies of patients are used in our analysis, defining a comparison of glomerular transcriptome profiles of adultonset steroid-sensitive focal segmental glomerulosclerosis and minimal change disease [16]. Transcriptomic and proteomic profiling, revealing insights of mesangial cell function in patients with IgA nephropathy [17]. Glomerular transcriptome from subjects in the NEPTUNE cohort [18]. Shared molecular targets in the glomerular transcriptome from patients with nephrotic syndrome and ANCA-associated vasculitis. Glomerular transcriptome from European renal cDNA bank subjects and living donors [19].

\section{A computational algorithm for gene adjustment}

The statistical methods used to detect DE genes were as moderated t-statistics for the microarray data based on a linear model fit; by fixing a P-value as 0.0001 , estimated as a prior probability that a gene is DE [20,21]. Then the semantic similarity computation was assumed based on Wang method [22], and using GOSemSim [23] package among gene products, based on the information's content (IC), and a directed acyclic graph (DAG) [24]. The IC-based measures depend on the frequencies of two GO terms involved on their closest common ancestor term in a specific corpus of GO annotations [25, 26]. The similarity computation was assumed for the three distinct aspects molecular functions (MF), biological processes (BP), and cellular components (CC). The best-match average strategy (BMA) was also used to calculate the average of all maximum similarities on each row and column for the similarity computation. Furthermore, a new scoring for the gene similarity measures for every pair of genes is demonstrated here (Formula. 1). Through which the matrix evaluates whether the means and normally distributed score within each independent pair of genes of samples evaluates an important significance or not by introducing the matrix-Similarity-based.

$$
M_{\text {simsc }}\left(g_{1}, g_{2}\right)=\sum_{n=1}^{\text {Lengthx }} \sum_{n=n+1}^{\text {Lengthx }}\left(\sqrt{\frac{1}{2} *\left(\frac{\delta_{1}^{2}}{\delta_{2}^{2}}+\frac{\delta_{2}^{2}}{\delta_{1}^{2}}\right)}\right) *\left(\frac{\mu_{1}+\mu_{2}}{2}\right)^{2}
$$

- Only the paired groups can be used to perform the paired test.

- The sample means are denoted as $\mu_{1}$ and $\mu_{2}$ for each score of similarity.

- Each score is sampled independently and randomly.

- The sample standard deviations $\delta_{1}$ and $\delta_{2}$ are normally distributed within each of the two rows.

Gene prediction results consist of a matrix-Expression-Similarity-Frequency-based as a new adjusted scoring for the DE measurement mixed with their scores and frequencies of occurrence of matrix-Similarity-based, which yield the final association score. These statistic scores are used for the candidate's genes as demonstrated here (Formula. 2). The genes with the highest scores will be selected and improved first then to serve as inputs to the machine learning steps. 


$$
M_{C o m b S c}=n_{\text {expr }} * M_{\text {expr }}+n_{\text {sel }} * M_{\text {simsc }}
$$

- A number of expressed genes provided with fixed P-value was combined to the expression matrix.

- A number of correlated genes combined to semantic similarity matrix.

\section{Prioritization of candidate genes based Machine Learning}

Machine learning techniques [27, 28, 29] were performed first by clustering our gene products; from the normalized final scores (results of Formula. 2), segmenting their into k clusters to understand biological processes as well as molecular functions $[30,31]$. Each cluster represents a group of similar observations performed using the ward method and Euclidean distance. Whereas, objects from different clusters are as dissimilar as possible. The algorithm can stop when the assignment of genes to clusters has no longer changed [32, 33]. Further, each genes cluster list has been exposed to the hgu133a,hgu133plus2 database of homo sapiens, as a direct mapping of gene symbol $[34,35]$, then implemented into a hypergeometric model to assess whether the number of selected genes are linked/associated with the pathogenesis of the diseases [36, 37]. Moreover, all enriched terms are associated with their enrichment scores, allowing the possibility to cross from high-level concepts to detailed pathway diagrams showing bio-molecular events using GroupGO(), EnrichGO() function $[38,39]$. Genetic variation was also studied, based on the genome-wide association study (GWAS) Catalog to tag variation across the genome, and enable investigations of causal variants [40, 41, 42, 43]. The most promising conserved non-coding regions of the promoters/enhancers [44] based oPOSSUM [45]; were also used to select the interaction between our gene products. Whose interactions between genes and transcription factors (TFs) are important for understanding gene regulation and the origin of protein complex components [46].

To facilitate the prioritization of causative genes as the second step of machine learning and based on supervised learning algorithm tools. A new gene prioritization algorithm tools were explained based on a logistic regression method [47, 48, 49, 50]. The gene prioritization boosts the power of identifying the most promising phenotype-associated among those clusters in normal conditions in different tissues (in which each gene can have a normalized score of expression in Tissue expression database [51]).

Score $_{\text {prioritization }}\left(\right.$ Gene $_{i} \mid$ Tissue $\left._{t}\right)=\mu_{0}+\mu_{1} X_{1}+\mu_{2} X_{2}+\mu_{3} X_{3}+\ldots+\mu_{n} X_{n}$

- $\mu_{0}$ is the means of expression-tissue-specific for a given gene with fixed tissues.

- $\mu_{1}, \mu_{2}, \mu_{3}, . . \mu_{n}$ represents the means of (frequencies of occurrence) for target genes for each process.

- X1, X2, X3,..Xn represents the normalized expression values of biological processes, GWAS, and TFs, etc. 
Following the hypothesis that genes underlying similar tissues will share functional and phenotype characteristics, we incorporate logistic regression for any given training genes which needed to be prioritized (formula. 3). The gene prioritization model could be formulated by arranging the candidate genes (i) in the order of their tissues (t) first; to be sure to be associated with the pathology. Our gene prioritization algorithm takes two inputs: a collection of evidence sources defining a phenotype/trait of interest, and enhancer/promoter interactions information, extracted for a given gene, linked to each other with a normalized score; reflecting the "likelihood" for each gene to be responsible for the phenotype. Then a second factor represents a prioritization mean factor (frequencies of occurrence of each entire). The output of the algorithm is a list of candidate genes arranged according to calculated scores for each tissue.

Overall, a full process of extracting transforming, and loading (ETL) data from homogeneous or heterogeneous sources $[52,53]$ was established to access our data. In short, it's an essential component in cleansing, customization, reformatting, integration, and insertion of our prerequired data $[54,55,56]$. In this paper, we tried to navigate through our adjusted genes to conceptualize the ETL processes, as shown in (Fig. 2); modeling a prioritization tool first, then modeling genetic interaction constructs into proper storage (format/structure) for querying and analysis.

\section{Genes Interaction}

Genetic interactions of omics data refer to a combination of pairs of genes into different tissues of fixed clusters as shown in (Fig. 3), whose contribution to a phenotype between specific variants in complex traits and tissues remains a major challenge $[57,58,59]$. Moreover, as the number of interacting genes increases, and because the traditional statistical methods are limited to their ability to identify interacting genes in high dimensions data [60,61,62]. We developed a novel algorithm-based model; called mixed-gene tissues interaction (MGTI) using our previous data. It first calculated gene prioritization scores and then identifies significantly interacted mixed-gene between the selected tissues using (formula. 4), reflecting strengths of regulatory interactions, aimed to understand etiology for our glomerular disease as a use case.

$$
\text { Score }_{\text {inter }}\left(g_{1}, g_{2}\right)=\frac{\log _{10}\left(S_{p}\left(g_{1}\right)\right)+\log _{10}\left(S_{p}\left(g_{2}\right)\right)}{2+\log _{10}\left(\max \left(S_{p}\left(g_{1}, g_{2}\right)\right)\right)}
$$

- $S_{p}$ represents a score of genes prioritization.

- The interaction scores are between $[0,1]$.

- When the interaction scores are $\cong[0.31,1]$, the MGTI model reflects an increased regulation of diseases.

- When the interaction scores are $\cong[0.1,0.3]$, the MGTI model reflects a decreased regulation of diseases, in which we should interact more with other possible genes.

\section{Results and Discussion}

The spectrum of glomerular diseases is an abnormal control of complement cascade activation, whose actions are considered as part of the innate immune system, 
procuring an immune complex deposition of fragments of C3 in glomeruli [63]. In most cases, GD is often resulting in damage to the kidneys, of which the cause is unknown. The first thing we do to measure the transcriptional levels was the validation of the information stored in raw data measurements [64, 65]. Where, the last was more described in our paper [66]; published of ACM organization, Proceedings of the New Challenges in Data Sciences: Acts of the Second Conference of the Moroccan Classification Society; as validation process of the information stored in expression sets. Each experiment was first analyzed separately, and the results have then combined based on their P-value [67].

\section{Genetic contributions and their statistical impact for estimation of the predictive models of gene}

Results of each number of DE genes were extracted, combined, and expected in a uniform distribution of the $\mathrm{P}$-value that corresponds to five different datasets (Table. 1). The semantic similarity results analysis is represented as the distance of (dis)/similarity between our list of genes in the interval of $[0,1]$. In which, when $\operatorname{Sim}\left(g_{i}, g_{j}\right)=1$ refers that $(i=j)$. Afterwards, when $\operatorname{Sim}\left(g_{i}, g_{j}\right) \cong[0.6,1]$ means that the precision of semantic similarities over genes in $G O\left(g_{i}, g_{j}\right)$ is more significant, and related to common pathologies. Then, when $\operatorname{Sim}\left(g_{i}, g_{j}\right) \cong[0,0.5]$ refers that the precision of semantic similarities over genes in $G O(i, j)$ can be significant or related to other common pathologies. However, many genes had a high score on the expression in parallel to a low score of GO (or the reverse). Whose the classical functional analysis in the literature is based only on the selection of genes from experiments, then to search their dis/similarity score. This problem was inspired us to combine/adjust both scores (expression and similarity) in a single formula, giving more chance to predict genes related to pathologies based on their occurrence frequencies. For that, we propose a novel gene selection method by introducing a novel matrix-Expression-Similarity-Frequency-based. Different threshold values give different levels of sensitivity and specificity, whether the low threshold (with red color) refers to a false positive, and the high threshold (with blue color) refers to true positive, as fixed for the validation of our study. This threshold makes it more likely specific (more high positives) against more sensitive (more low positives); as shown in the figure (Fig. 4) with a given fixed threshold of observations.

\section{Evaluation/Prioritization based tissues using regression model}

An estimating results score from formula. 2 for each gene was the input for computing the clustering algorithms. Four clusters were found, each cluster represents a group of similar observations. To search for shared functions, all gene products were linked to different databases as an enrichment, traits (non-coding variants), tissues databases, and conserved transcription factor binding sites selected based on their score of \%GC content. These kinds of analyses generate a huge quantity of data, which needs to be given a biological interpretation. Majors of these genes fall under (kidney, blood, urine, immune system, and embryonic) tissues, and in the hypothesis interpretation and validation section for the GD, we were based on a specific entire to generate a list of maximum information of genes related to a specifics biological pathway terms as regulation of inflammatory response, regulation of acute inflammatory response, regulation of protein processing/maturation, positive regulation 
of glomerulus development, regulation of glomerular mesangial cell proliferation, regulation of adaptive immune response, complement activation, etc. A circular (Circos) plot (Fig. 6) was the most efficient to visualize genomic data giving us the possibility to easily map information on a single plot. A Heatmap-like functional classification plot (Fig. 5), was used to visualize the most significant terms with the terms expected from the literature, according to some scores for the causative genes as (EGR1, IL33, BMP2, SLAMF8, etc.). In which, we filtered and selected the most dominant GO terms according to GD that include kidney vasculature development, regulation of cell activation/ inflammatory/immune effector/adaptive immune/glomerulus/glomerular mesangial cell proliferation] development, etc. A barplot (Fig. 5) was used to visualize the gene-variant-trait associations among GWAS catalog for the most dominant terms like complement C3, C4, C7 measurement, serum IgE/IgA measurement, c-reactive protein measurement, nephrotic syndrome, immune system disease, tuberculosis, glomerular filtration rate, chronic kidney disease, urinary metabolite measurement, C-reactive protein measurement, glomerular filtration rate, etc. In addition, many genes such as TNXA, FCER1A, NME3, FMOD, BTG2, PTGER4, AXL, CYP1A2, CYTL1, BHLHE40, IFI16, SPON1, ETNPPL, COL14A1, ITGAV, MYOZ2, CAMK2A, SORT1, RANBP1, etc. represented some trait association. Finally, the PieChart plot (Fig. 5) represented the means of expression of genes in the selected tissues; Kidney, Kidney cancer cell, Immune system, Urine, Blood, Blood vessel, Blood plasma, Hematopoietic stem cell, Parenchyma, Uroepithelium, HEK 293 EBNA cell, HEK 293ET cell, HK 2 cell.

The Gene prioritization model could be formulated as follows (which is composed of two parts): arrange candidate genes in the order of their normalized scores and frequencies of occurrence from formula. 2, then to their normalized scores and frequencies of occurrence for categorical tissues, biological process, TFs, and variants. By the same token, the logistic regression model (parametric regression) was performed for categorical data to prioritize the dependent variable using a given set of independent variables for solving Classification problems. In logistic regression, it is not necessary to have a linear relationship between the dependent and independent variable, but there should not be collinearity between the independent variable. As discussed above, logistic regression was used to classify elements of each cluster under various tissues by calculating the mean of normalized expression of each element of the set. A confidence regression line provides a representation of the uncertainty in a sense, we can prioritize among a cluster of genes, the most promising ones based on their prioritization scores on each selected tissue as visualized on (Fig. 7). We notice also in the table (Table. 2), that many redundant genes have been more promising by our applying the priority model for fixed tissues, this has only one interpretation which has a great value of the expression in the different tissue analyzed. The process of arranging all possible prioritizing genes causing disease based on their logistic regression has shown consistent genes may reside in distinct pathways and affect the promoter/regulatory region of location-related, organismspecific.

Based PubMed resource, for information retrieval, summarizing and comparing topics according to their frequencies of occurrence $[68,69,70]$, to broaden or/and 
narrow a search [71], and to exclude unwanted search terms/concepts from a specific speech as "glomerulopathies", "diabetic kidney disease", "tumor Nephrectomy", "diabetic nephropathy", " focal segmental glomerulosclerosis ", "rapidly progressive glomerulonephritis", "minimal change disease", and "membranous glomerulonephritis". In addition, 972 abstracts that were extracted, had 11 genes as COL4A5, EGR1, GDF15, CPE, CASK, NT5E, JUN, AXL, CCL3, IL33, ITGAV from our candidate's genes have already been reported in previous studies with GD.

\section{Genetic interactions bridging transcription factor and pathways in genome-wide association studies}

Traditional statistical methods consider gene-gene interactions or estimate interactions among only a small number of phenotype information with significant main effects. However, our MGTI algorithm-based model can be applied especially when the data are high dimensional (many attributes or independent variables) or when interactions between more than two tissues can play a role in the analysis of human disease etiology and regulation data mining. To perform ETL (extract-transformload) operations, either a vector of SQL commands was used to select data based on a specific entity. Here we were focused to select the best score of gene-gene interaction to support and validate the hypothesis validation. Representative (Table. 3 and 4) have been added to show some randomly gene-gene interaction-algorithm tools based MGTI model. Finally, the gene interaction results encode a superfamily of proteins, playing a role in transcriptional expression. Whether ligands of this family bind various enzyme binding and receptors/initiators; leading to recruitment and activation of family transcription and/or signaling factors that regulate the level and stability of gene expression. The encoded proteins possess an intracellular and/or extracellular domain that is composed of different motifs. This gene may be involved in several cellular functions including multiple cell types and various tissues. Alternative splicing results in multiple transcript variants of these candidate genes.

The early diagnosis and prognosis of any disease type have more necessity of using of bioinformatics tools. As it can facilitate the subsequent clinical management of patients. This follow-up analysis can be applied also to other types of data such as cancer data, etc. Finally, our global objective was to incorporate/develop a Shiny web-based application as an $\mathrm{R}$ framework designed to help and facilitate users to navigate under "Shiny apps" as tutorials, about us, and test our algorithms tools: gene prediction, prioritization, and interaction.

\section{Conclusions}

The linked resources of biological/clinical data-based expression profiles ( adjusted score), were used to validate molecular biology research. Moreover, the result of the highest expression is in most cases; a list of genes, defining a comparison of glomerular transcriptome profiles established to investigate the underlying disease mechanisms; as a representative knowledge of focal segmental glomerulosclerosis, minimal change disease, insights into mesangial cell function in patients with IgA nephropathy. Majors of our prioritized genes fall under the transcription co-factor 
binding, regulation of glomerular mesangial cell proliferation, regulation of adaptive immune response, complement activation, etc. Furthermore, that achieves and validates the target of the best-represented gene-gene interactions findings.

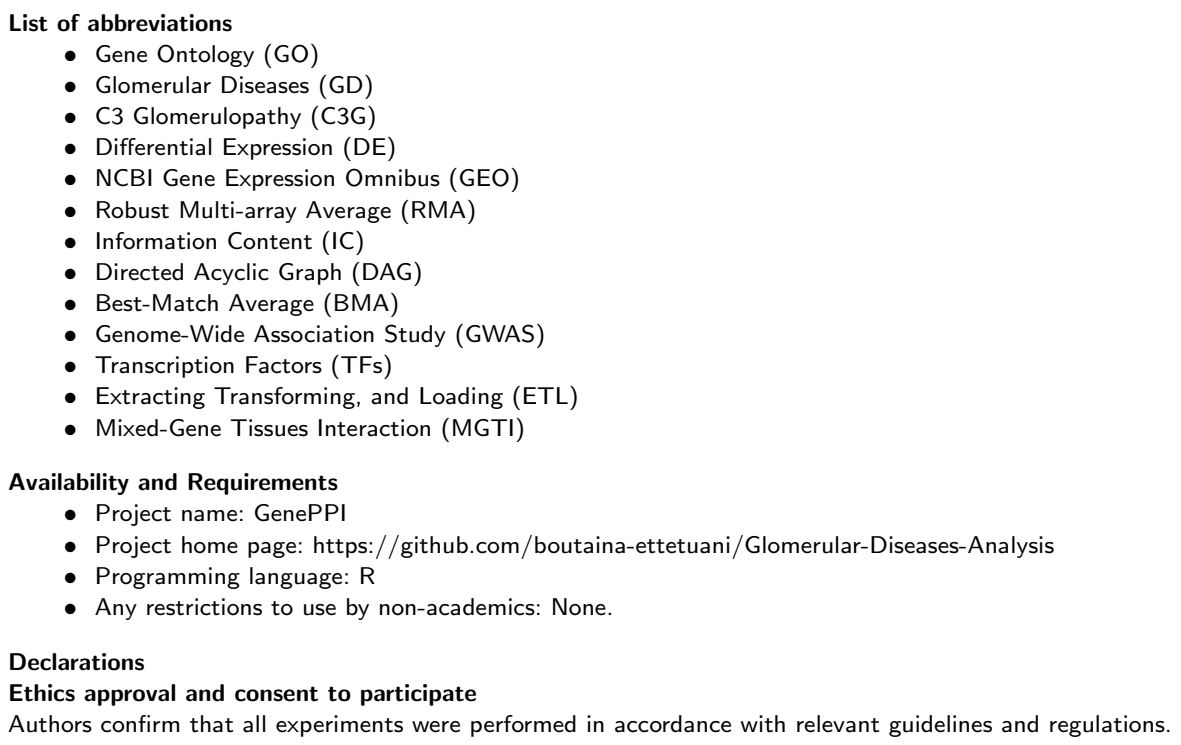

Availability of data and materials

All raw data accession are accessible via the public repository of microarray data NCBI Gene Expression Omnibus: (www.ncbi.nlm.nih.gov/geo/query/acc.cgi), with an accession: (GSE69814, GSE93798, GSE108113, GSE104948), in which the data input expected during the current GenePPI project after applying our algorithms are included in the R Shiny application, and linked as available in the GitHub repository:

https://github.com/boutaina-ettetuani/Glomerular-Diseases-Analysis, in which the data supporting can be found as CSV files.

\section{Competing interests}

The authors declare that they have no competing interests.

Funding

No funding was obtained for this study.

Author's contributions

BE contributed to R-scripting for the development of GenePPI structural analyses, statistics, implementation of the method. All authors have read and approved the final manuscript including results, figures shaping, manuscript formatting, and manuscript writing. RC contributed to the interpretation of results. AM contributed to the interpretation implementation of the method, and structural analysis. AM and RC contributed equally in literature research.

\section{Acknowledgements}

BE would like to acknowledge and thank Prof. Dr. AM and Prof. Dr. RC for their supervision and support in this work.

Author details

${ }^{1}$ Systems and Data Engineering Team, National School of Applied Sciences, Abdelmalek Essaadi University, Tangier, MOROCCO. ${ }^{2}$ Life and Health Sciences Team, Faculty of Medicine and Pharmacy, Abdelmalek Essaadi University, Tangier, MOROCCO.

References

1. Riedl, M., Thorner, P., Licht, C.: C3 glomerulopathy. Pediatric Nephrology 32(1), 43-57 (2017)

2. Cook, H.T., Pickering, M.C.: Histopathology of mpgn and c3 glomerulopathies. Nature Reviews Nephrology 11(1), 14 (2015)

3. Coelho, S.S., Fernandes, A.R., Soares, E., Valério, P., Matos, B., Romão, H., Góis, M., Sousa, H., Fidalgo, T., Natário, A.S., et al.: A new complement factor b mutation associated with crescentic c3 glomerulopathy; a case report. Journal of Nephropathology 8(3) (2019)

4. Iatropoulos, P., Noris, M., Mele, C., Piras, R., Valoti, E., Bresin, E., Curreri, M., Mondo, E., Zito, A., Gamba, S., et al.: Complement gene variants determine the risk of immunoglobulin-associated mpgn and c3 glomerulopathy and predict long-term renal outcome. Molecular immunology 71, 131-142 (2016) 
5. Xiao, X., Pickering, M.C., Smith, R.J.: C3 glomerulopathy: the genetic and clinical findings in dense deposit disease and c3 glomerulonephritis. In: Seminars in Thrombosis and Hemostasis, vol. 40, pp. 465-471 (2014). Thieme Medical Publishers

6. Tsai, H.-M., Rice, L., Sarode, R., Chow, T.W., Moake, J.L.: Antibody inhibitors to von willebrand factor metalloproteinase and increased binding of von willebrand factor to platelets in ticlopidine-associated thrombotic thrombocytopenic purpura. Annals of internal medicine 132(10), 794-799 (2000)

7. Rodriguez de Cordoba, S., Subías, M., Pinto, S., Tortajada, A.: Genetics of atypical hemolytic uremic syndrome (ahus) (2014)

8. Legendre, C.M., Licht, C., Muus, P., Greenbaum, L., Babu, S., Bedrosian, C., Bingham, C., Cohen, D., Delmas, Y., Douglas, K., et al.: Terminal complement inhibitor eculizumab in atypical hemolytic-uremic syndrome. New England Journal of Medicine 368(23), 2169-2181 (2013)

9. Klaus, B., Reisenauer, S.: An end to end workflow for differential gene expression using affymetrix microarrays. F1000Research 5 (2016)

10. Clough, E., Barrett, T.: The gene expression omnibus database. In: Statistical Genomics, pp. 93-110. Springer, ??? (2016)

11. McCall, M.N., Irizarry, R.A.: Thawing frozen robust multi-array analysis (frma). BMC bioinformatics 12(1), 1-7 (2011)

12. Okoniewski, M.J., Miller, C.J.: Hybridization interactions between probesets in short oligo microarrays lead to spurious correlations. BMC bioinformatics 7(1), 1-14 (2006)

13. Sabir, B., Ullah, F., Babar, M.A., Gaire, R.: Machine learning for detecting data exfiltration: A review. ACM Computing Surveys (CSUR) 54(3), 1-47 (2021)

14. $\mathrm{Xu}, \mathrm{C}$., Jackson, S.A.: Machine learning and complex biological data. Springer (2019)

15. Tarca, A.L., Carey, V.J., Chen, X.-w., Romero, R., Drăghici, S.: Machine learning and its applications to biology. PLoS computational biology 3(6), 116 (2007)

16. Tong, J., Xie, J., Ren, H., Liu, J., Zhang, W., Wei, C., Xu, J., Zhang, W., Li, X., Wang, W., et al.: Comparison of glomerular transcriptome profiles of adult-onset steroid sensitive focal segmental glomerulosclerosis and minimal change disease. PLoS One 10(11), 0140453 (2015)

17. Liu, P., Lassén, E., Nair, V., Berthier, C.C., Suguro, M., Sihlbom, C., Kretzler, M., Betsholtz, C., Haraldsson, B., Ju, W., et al.: Transcriptomic and proteomic profiling provides insight into mesangial cell function in iga nephropathy. Journal of the American Society of Nephrology 28(10), 2961-2972 (2017)

18. Mitrofanova, A., Molina, J., Santos, J.V., Guzman, J., Morales, X.A., Ducasa, G.M., Bryn, J., Sloan, A., Volosenco, I., Kim, J.-J., et al.: Hydroxypropyl- $\beta$-cyclodextrin protects from kidney disease in experimental alport syndrome and focal segmental glomerulosclerosis. Kidney international 94(6), 1151-1159 (2018)

19. Grayson, P.C., Eddy, S., Taroni, J.N., Lightfoot, Y.L., Mariani, L., Parikh, H., Lindenmeyer, M.T., Ju, W., Greene, C.S., Godfrey, B., et al.: Metabolic pathways and immunometabolism in rare kidney diseases. Annals of the rheumatic diseases $77(8), 1226-1233$ (2018)

20. Jeffery, I.B., Higgins, D.G., Culhane, A.C.: Comparison and evaluation of methods for generating differentially expressed gene lists from microarray data. BMC bioinformatics 7(1), 1-16 (2006)

21. Sartor, M.A., Tomlinson, C.R., Wesselkamper, S.C., Sivaganesan, S., Leikauf, G.D., Medvedovic, M.: Intensity-based hierarchical bayes method improves testing for differentially expressed genes in microarray experiments. BMC bioinformatics 7(1), 1-17 (2006)

22. Wang, J.Z., Du, Z., Payattakool, R., Yu, P.S., Chen, C.-F.: A new method to measure the semantic similarity of go terms. Bioinformatics 23(10), 1274-1281 (2007)

23. Yu, G., Li, F., Qin, Y., Bo, X., Wu, Y., Wang, S.: Gosemsim: an r package for measuring semantic similarity among go terms and gene products. Bioinformatics 26(7), 976-978 (2010)

24. Mazandu, G.K., Mulder, N.J.: Information content-based gene ontology semantic similarity approaches: toward a unified framework theory. BioMed research international 2013 (2013)

25. Thomas, P.D.: The gene ontology and the meaning of biological function. In: The Gene Ontology Handbook, pp. 15-24. Humana Press, New York, NY, ??? (2017)

26. Gaudet, P., Škunca, N., Hu, J.C., Dessimoz, C.: Primer on the gene ontology. In: The Gene Ontology Handbook, pp. 25-37. Humana Press, New York, NY, ??? (2017)

27. Ke, P.-f., Xiong, D.-s., Li, J.-h., Pan, J., et al.: An integrated machine learning framework for a discriminative analysis of schizophrenia using multi-biological data. Scientific reports 11(1), 1-11 (2021)

28. Ganggayah, M.D., Taib, N.A., Har, Y.C., Lio, P., Dhillon, S.K.: Predicting factors for survival of breast cancer patients using machine learning techniques. BMC medical informatics and decision making 19(1), 1-17 (2019)

29. Zhang, X.-D.: Machine learning. In: A Matrix Algebra Approach to Artificial Intelligence, pp. $223-440$. Springer, ??? (2020)

30. Maulik, U., Mukhopadhyay, A., Bandyopadhyay, S.: Combining pareto-optimal clusters using supervised learning for identifying co-expressed genes. BMC bioinformatics 10(1), 1-16 (2009)

31. Chou, J.W., Zhou, T., Kaufmann, W.K., Paules, R.S., Bushel, P.R.: Extracting gene expression patterns and identifying co-expressed genes from microarray data reveals biologically responsive processes. BMC bioinformatics 8(1), 1-16 (2007)

32. de Winter, J.C., Gosling, S.D., Potter, J.: Comparing the pearson and spearman correlation coefficients across distributions and sample sizes: A tutorial using simulations and empirical data. Psychological methods 21(3), 273 (2016)

33. Gry, M., Rimini, R., Strömberg, S., Asplund, A., Pontén, F., Uhlén, M., Nilsson, P.: Correlations between rna and protein expression profiles in 23 human cell lines. BMC genomics 10(1), 1-14 (2009)

34. Yu, G.: Statistical analysis and visualization of functional profiles for genes and gene clusters. Journal of Integrative Biology 16(5), 284-287 (2012)

35. Smedley, D., Haider, S., Ballester, B., Holland, R., London, D., Thorisson, G., Kasprzyk, A.: Biomart-biological queries made easy. BMC genomics 10(1), 1-12 (2009)

36. Yu, G.: Reactome pathway analysis. Homo 1266738(1266738), 29 (2012) 
37. Fabregat, A., Jupe, S., Matthews, L., Sidiropoulos, K., Gillespie, M., Garapati, P., Haw, R., Jassal, B., Korninger, F., May, B., et al.: The reactome pathway knowledgebase. Nucleic acids research 46(D1), 649-655 (2018)

38. Yu, G., Wang, L.-G., Dall'Olio, G., Yu, M.G.: Package 'clusterprofiler' (2018)

39. Sidiropoulos, K., Viteri, G., Sevilla, C., Jupe, S., Webber, M., Orlic-Milacic, M., Jassal, B., May, B., Shamovsky, V., Duenas, C., et al:: Reactome enhanced pathway visualization. Bioinformatics 33(21), 3461-3467 (2017)

40. Johnson, R.C., Nelson, G.W., Troyer, J.L., Lautenberger, J.A., Kessing, B.D., Winkler, C.A., O'Brien, S.J.: Accounting for multiple comparisons in a genome-wide association study (gwas). BMC genomics 11(1), 1-6 (2010)

41. Scharf, J.M., Yu, D., Mathews, C.A., Neale, B.M., Stewart, S.E., Fagerness, J.A., Evans, P., Gamazon, E., Edlund, C.K., Service, S., et al.: Genome-wide association study of tourette's syndrome. Molecular psychiatry 18(6), 721-728 (2013)

42. Garfield, V.: Sleep duration: A review of genome-wide association studies (gwas) in adults from 2007 to 2020. Sleep Medicine Reviews, 101413 (2020)

43. Butler, J.M., Hall, N., Narendran, N., Yang, Y.C., Paraoan, L.: Identification of candidate protective variants for common diseases and evaluation of their protective potential. BMC genomics 18(1), 1-11 (2017)

44. Zolotareva, O., Kleine, M.: A survey of gene prioritization tools for mendelian and complex human diseases. Journal of integrative bioinformatics 16(4) (2019)

45. Ho Sui, S.J., Fulton, D.L., Arenillas, D.J., Kwon, A.T., Wasserman, W.W.: opossum: integrated tools for analysis of regulatory motif over-representation. Nucleic acids research 35(suppl_2), 245-252 (2007)

46. Suravajhala, P., Benso, A.: Prioritizing single-nucleotide polymorphisms and variants associated with clinical mastitis. Advances and applications in bioinformatics and chemistry: AABC 10, 57 (2017)

47. Zhang, K., Geng, W., Zhang, S.: Network-based logistic regression integration method for biomarker identification. BMC systems biology 12(9), 113-122 (2018)

48. Lee, H.-C., Yoon, S.B., Yang, S.-M., Kim, W.H., Ryu, H.-G., Jung, C.-W., Suh, K.-S., Lee, K.H.: Prediction of acute kidney injury after liver transplantation: machine learning approaches vs. logistic regression model. Journal of clinical medicine 7(11), 428 (2018)

49. Christodoulou, E., Ma, J., Collins, G.S., Steyerberg, E.W., Verbakel, J.Y., Van Calster, B.: A systematic review shows no performance benefit of machine learning over logistic regression for clinical prediction models. Journal of clinical epidemiology 110, 12-22 (2019)

50. Nusinovici, S., Tham, Y.C., Yan, M.Y.C., Ting, D.S.W., Li, J., Sabanayagam, C., Wong, T.Y., Cheng, C.-Y.: Logistic regression was as good as machine learning for predicting major chronic diseases. Journal of clinical epidemiology 122, 56-69 (2020)

51. Palasca, O., Santos, A., Stolte, C., Gorodkin, J., Jensen, L.J.: Tissues 2.0: an integrative web resource on mammalian tissue expression. Database 2018 (2018)

52. Biswas, N., Sarkar, A., Mondal, K.C.: Efficient incremental loading in etl processing for real-time data integration. Innovations in Systems and Software Engineering 16(1), 53-61 (2020)

53. Biswas, N., Sarkar, A., Mondal, K.C.: Empirical analysis of programmable etl tools. In: International Conference on Computational Intelligence, Communications, and Business Analytics, pp. 267-277 (2018). Springer

54. Greiff, V., Miho, E., Menzel, U., Reddy, S.T.: Bioinformatic and statistical analysis of adaptive immune repertoires. Trends in immunology 36(11), 738-749 (2015)

55. Tecnico, R.: Etls for importing ncbi entrez gene, mirbase, mircancer and microrna into a bioinformatics graph database (2015)

56. Tecnico, R.: Etls for importing uniprotkb, hgnc, and reactome into a bioinformatics graph database

57. Subramanian, I., Verma, S., Kumar, S., Jere, A., Anamika, K.: Multi-omics data integration, interpretation, and its application. Bioinformatics and biology insights 14, 1177932219899051 (2020)

58. Gomez-Cabrero, D., Abugessaisa, I., Maier, D., Teschendorff, A., Merkenschlager, M., Gisel, A., Ballestar, E., Bongcam-Rudloff, E., Conesa, A., Tegnér, J.: Data integration in the era of omics: current and future challenges. BMC systems biology 8(2), 1-10 (2014)

59. Vasaikar, S.V., Straub, P., Wang, J., Zhang, B.: Linkedomics: analyzing multi-omics data within and across 32 cancer types. Nucleic acids research 46(D1), 956-963 (2018)

60. Yang, P., Ho, J.W., Yang, Y.H., Zhou, B.B.: Gene-gene interaction filtering with ensemble of filters. BMC bioinformatics 12(1), 1-10 (2011)

61. Gordon, D.E., Watson, A., Roguev, A., Zheng, S., Jang, G.M., Kane, J., Xu, J., Guo, J.Z., Stevenson, E., Swaney, D.L., et al.: A quantitative genetic interaction map of hiv infection. Molecular cell 78(2), 197-209 (2020)

62. Sun, H., Guo, Y., Lan, X., Jia, J., Cai, X., Zhang, G., Xie, J., Liang, Q., Li, Y., Yu, G.: Phenomodifier: a genetic modifier database for elucidating the genetic basis of human phenotypic variation. Nucleic acids research 48(D1), 977-982 (2020)

63. Pickering, M.C., D'agati, V.D., Nester, C.M., Smith, R.J., Haas, M., Appel, G.B., Alpers, C.E., Bajema, I.M., Bedrosian, C., Braun, M., et al.: C3 glomerulopathy: consensus report. Kidney international 84(6), 1079-1089 (2013)

64. Dalman, M.R., Deeter, A., Nimishakavi, G., Duan, Z.-H.: Fold change and p-value cutoffs significantly alter microarray interpretations. In: BMC Bioinformatics, vol. 13, pp. 1-4 (2012). BioMed Central

65. Kharchenko, P.V., Silberstein, L., Scadden, D.T.: Bayesian approach to single-cell differential expression analysis. Nature methods 11(7), 740-742 (2014)

66. Ettetuani, B., Chahboune, R., Moussa, A.: Meta-analysis for a therapeutic target involved in the activation of the genes associated with c3 glomerulopathy. In: Proceedings of the New Challenges in Data Sciences: Acts of the Second Conference of the Moroccan Classification Society, pp. 1-6 (2019)

67. Walsh, C.J., Hu, P., Batt, J., Santos, C.C.D.: Microarray meta-analysis and cross-platform normalization integrative genomics for robust biomarker discovery. Microarrays 4(3), 389-406 (2015)

68. Lin, J., Wilbur, W.J.: Pubmed related articles: a probabilistic topic-based model for content similarity. BMC 
bioinformatics 8(1), 1-14 (2007)

69. Rani, J., Ramachandran, S., et al.: pubmed. miner: An $r$ package with text-mining algorithms to analyse pubmed abstracts. Journal of biosciences 40(4), 671-682 (2015)

70. Gusenbauer, M., Haddaway, N.R.: Which academic search systems are suitable for systematic reviews or meta-analyses? evaluating retrieval qualities of google scholar, pubmed, and 26 other resources. Research synthesis methods 11(2), 181-217 (2020)

71. Kovalchik, S.: Rismed: download content from ncbi databases. R package version 2(5) (2015)

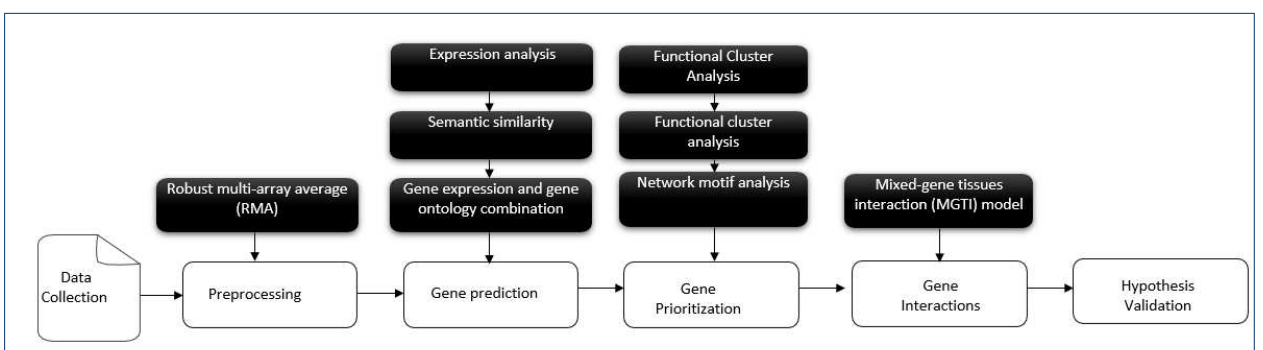

Figure 1 An overview of schematic workflow of the developed approach, consisting of five components: transcriptomics data collection and pre-processing steps first, then, gene prediction, and prioritization. After all, a genetic interactions model on a human genome-scale, then a hypothesis evaluation and validation.

Tables

Table 1 Description of the five datasets, in which all types of data was transcription profiling by array, with their fixed P-value when annotated to the human databases, and selected matrix-Expression-Similarity-Frequency-based.

\begin{tabular}{lllll} 
Id & Statut & $\begin{array}{l}\text { number } \\
\text { of samples }\end{array}$ & $\begin{array}{l}\text { number } \\
\text { of genes }\end{array}$ & Selected genes \\
\hline E-GEOD-69814 & 4-Jan-17 & 11 & 32321 & 71 \\
E-GEOD-93798 & 3-Jul-17 & 42 & 54675 & 34 \\
GSE108113/E-GEOD-104066 & 26-Jun-19 & 76 & 1416100 & 53 \\
GSE108113/E-GEOD-108109 & 17-Jul-18 & 111 & 1416100 & 68 \\
E-GEOD-104948 & 24-Jan-18 & 196 & 76958 & 183 \\
Total of genes & & & & 415 \\
Not duplicated & & & 412 \\
Annotated & & & & 209 \\
Selected & & & 100 \\
\hline
\end{tabular}




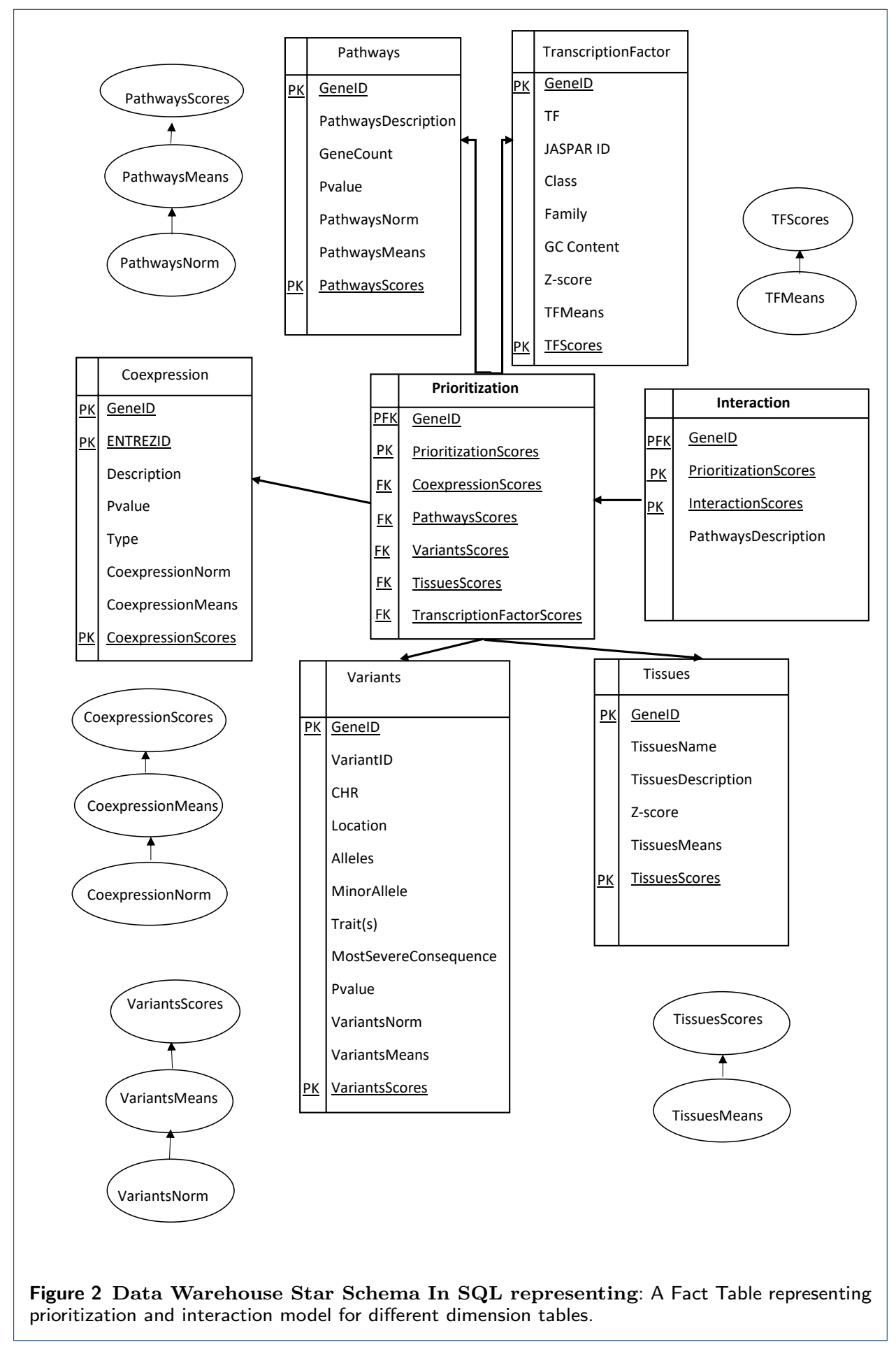




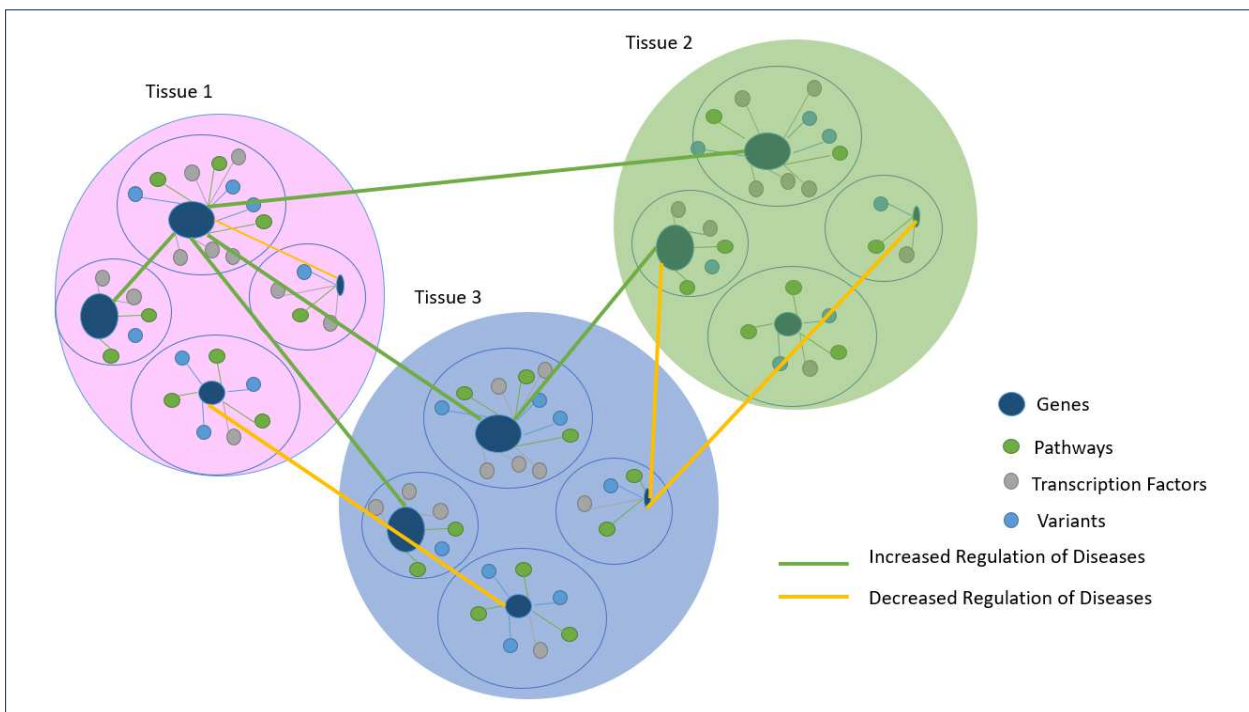

Figure 3 Genes prioritization results based tissues linked to each other by regulation of diseases links.

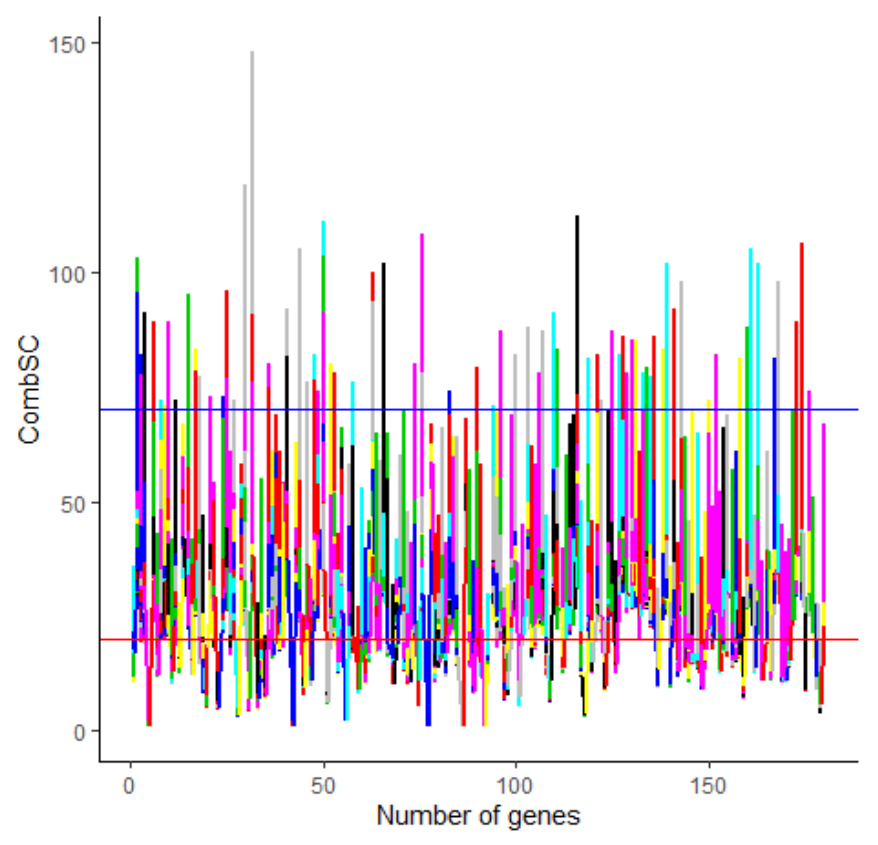

Figure 4 Visualization methods: Adjusted expression and similarity scores (based P3), and their frequency positions with a fixed low vs. high threshold as strategy used to valid the matrix-Expression-Similarity-Frequency-based. 


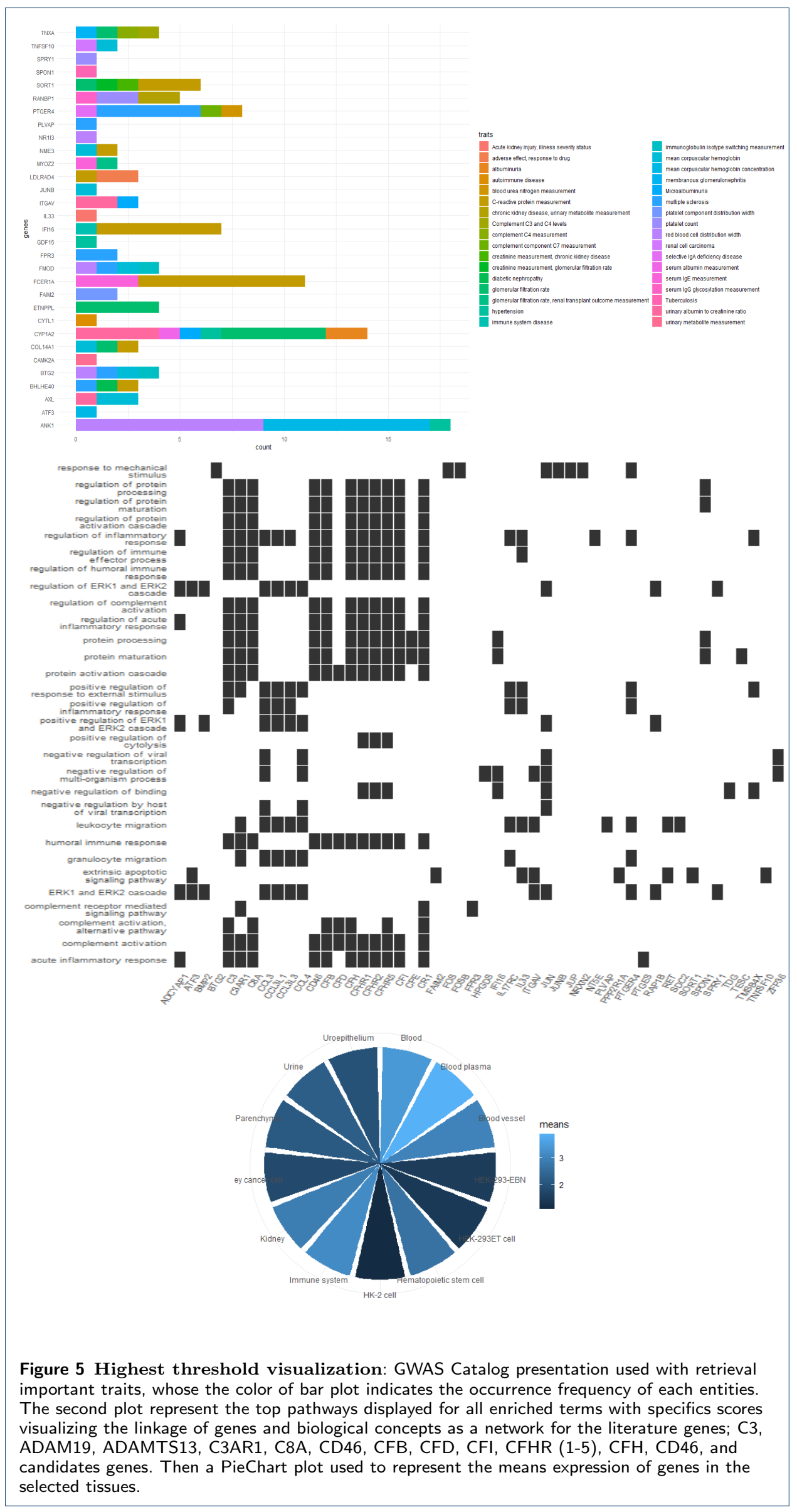




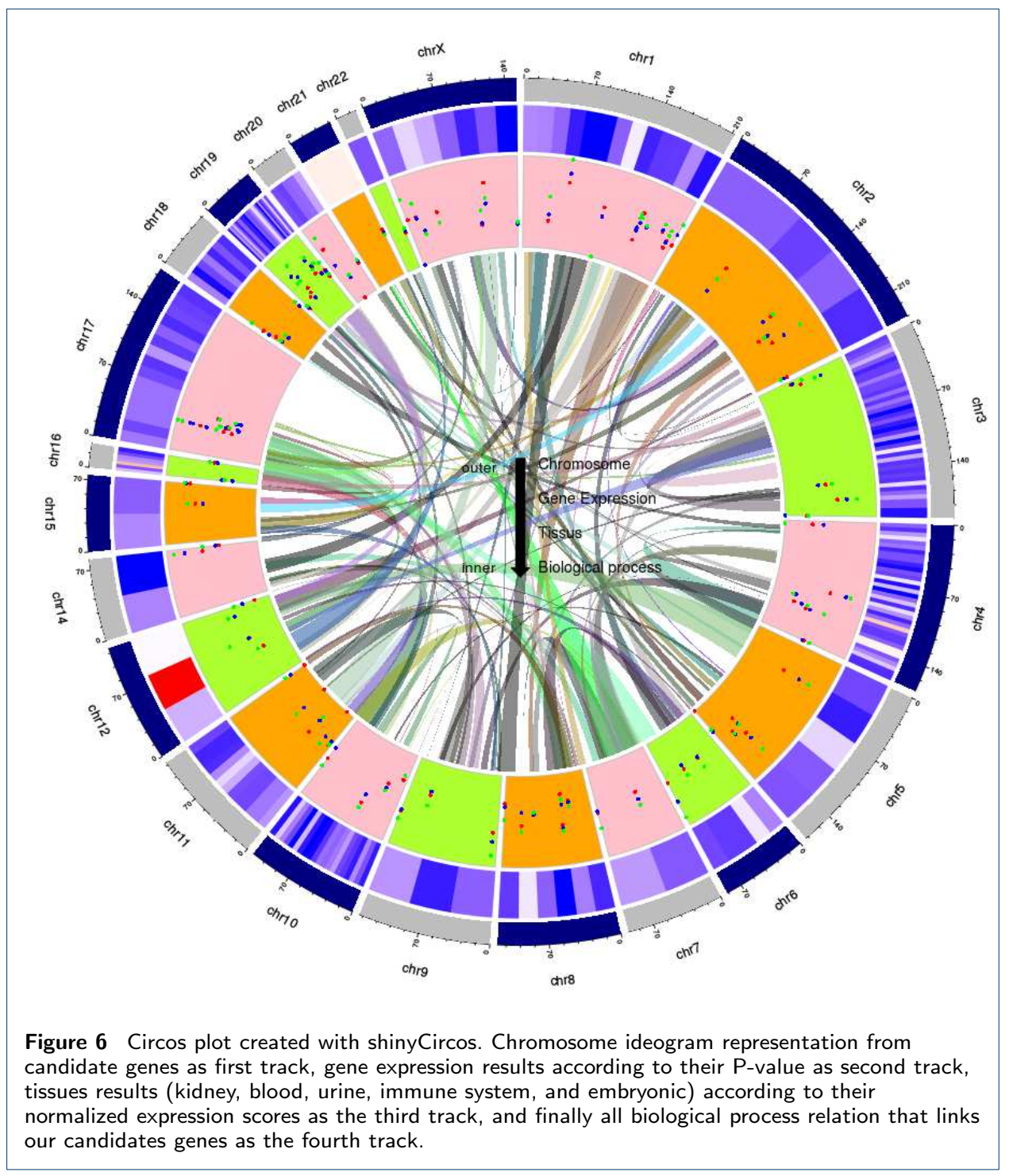




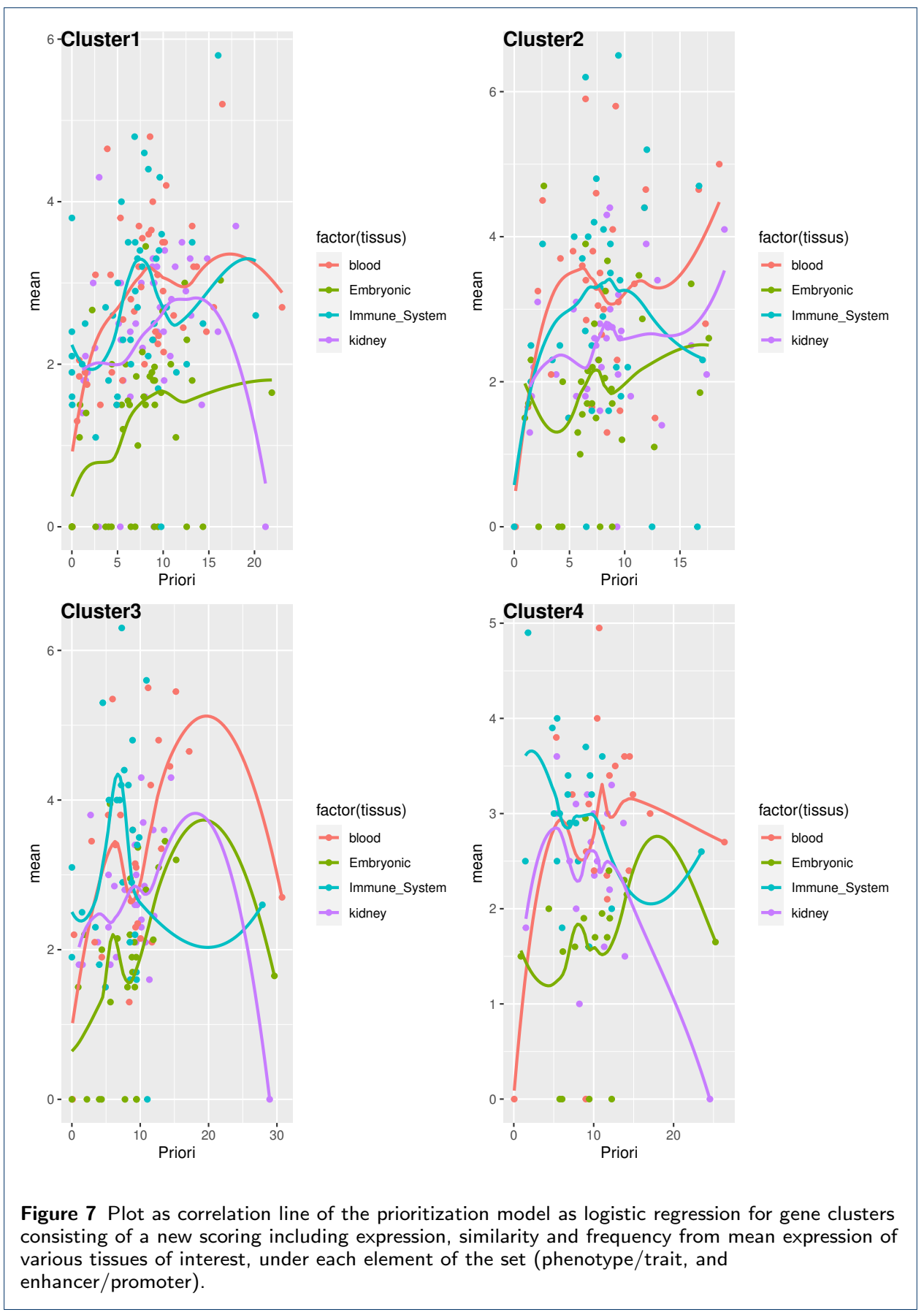


Table 2 The most promising genetic head data prioritization-model-based for the four clusters

\begin{tabular}{|lllll|}
\hline Cluster N 1 & & & & \\
\hline kidney & urine & Immune system & blood & Embryonic dev \\
\hline IL33 & SPON1 & SPON1 & SPON1 & SPON1 \\
RANBP1 & IL33 & IL33 & IL33 & IL33 \\
ANK1 & RANBP1 & RANBP1 & RANBP1 & RANBP1 \\
MYOZ2 & ANK1 & ANK1 & MYOZ2 & ANK1 \\
MEOX1 & MYOZ2 & MYOZ2 & PALB2 & PALB2 \\
CACNA1G & MEOX1 & MEOX1 & CACNA1G & MYOZ2 \\
PALB2 & CACNA1G & CACNA1G & ANK1 & MEOX1 \\
\hline Cluster N 2 & & & & \\
\hline kidney & urine & Immune system & blood & Embryonic dev \\
\hline BMP2 & BMP2 & COL15A1 & BMP2 & BMP2 \\
COL15A1 & COL15A1 & ITGAV & COL15A1 & COL15A1 \\
ITGAV & ITGAV & BMP2 & ITGAV & ITGAV \\
ATP13A3 & ATP13A3 & ATP13A3 & ATP13A3 & ATP13A3 \\
TNFSF10 & TNFSF10 & TNFSF10 & TNFSF10 & TNFSF10 \\
FGF7 & FGF7 & FGF7 & FGF7 & FGF7 \\
\hline Cluster N 3 & & & & \\
\hline kidney & Urine & Immune system & Blood & Embryonic dev \\
\hline SPON1 & SPON1 & SPON1 & SPON1 & SPON1 \\
RET & RET & RET & RET & RET \\
FOS & FOS & FOS & FOS & FOS \\
SFRP4 & SFRP4 & SFRP4 & AXL & AXL \\
AXL & NME3 & P2RY14 & SFRP4 & SFRP4 \\
NME3 & CA10 & NME3 & GDF15 & GDF15 \\
CA10 & BTG2 & CA10 & NME3 & CCL4 \\
\hline Cluster N 4 & & & & \\
\hline kidney & urine & Immune system & blood & Embryonic dev \\
\hline SPON1 & SPON1 & SPON1 & SPON1 & SPON1 \\
MYOZ2 & MYOZ2 & MYOZ2 & TDG & TDG \\
PALB2 & PALB2 & PALB2 & PALB2 & PALB2 \\
SORT1 & RAP1B & RAP1B & SPRY1 & MYOZ2 \\
RAP1B & NME3 & P2RY14 & MYOZ2 & SORT1 \\
TDG & SORT1 & NME3 & RAP1B & SPRY1 \\
\hline
\end{tabular}


Table 3 Global summary of mixed-gene tissues interaction (MGTI) model based on gene prioritization results as well as randomly selected genes from the results.

\begin{tabular}{|c|c|c|}
\hline \multicolumn{3}{|l|}{ Cluster N 1} \\
\hline & Genes 1: IL33 & Genes 2: RANBP1 \\
\hline Mean-coExp(adjusted) & 0.17 & IL33 \\
\hline Mean-tissues & 5.8 & SPON1 \\
\hline Description-tissues & "Immune system" & "Kidney" \\
\hline Mean-BP & 0.59 & - \\
\hline Description-BP & $\begin{array}{l}\text { "regulation of inflammatory response" } \\
\text { "regulation of immune effector process" } \\
\text { "positive regulation of response to } \\
\text { external stimulus" } \\
\text { "positive regulation of cytokine } \\
\text { production" } \\
\text { "positive regulation of inflammatory } \\
\text { response" } \\
\text { "adaptive immune response based } \\
\text { on somatic recombination } \\
\text { of immune receptors built } \\
\text { from immunoglobulin superfamily domains" } \\
\text { "positive regulation of proteolysis" } \\
\text { "regulation of adaptive immune response" } \\
\text { "regulation of neuroinflammatory response" } \\
\text { "positive regulation of cell activation" }\end{array}$ & - \\
\hline Mean-TFs & 0.77 & 1.54 \\
\hline Description-TFs & $\begin{array}{l}\text { "Myc", "CEBPA", " USF1", "TBP", } \\
\text { "FOXO3", "Arnt::Ahr", "Sox5" }\end{array}$ & $\begin{array}{l}\text { "Zfx", "MYC::MAX", } \\
\text { "Mycn", "RXRA::VDR", } \\
\text { "ZNF354C", "Myc", "Egr1" }\end{array}$ \\
\hline Mean-GWAS & 4.75 & 0.4 \\
\hline Description-GWAS & "Acute kidney injury" & $\begin{array}{l}\text { "urinary metabolite } \\
\text { measurement" } \\
\text { "chronic kidney disease" } \\
\text { "blood protein measurement" } \\
\text { "platelet count" } \\
\text { "serum IgG glycosylation } \\
\text { measurement" }\end{array}$ \\
\hline prioritization score & 16.53 & 16 \\
\hline \multicolumn{3}{|l|}{ Interaction score: 0.75} \\
\hline & Genes 1: SPON1 & Genes 2: CYP1A2 \\
\hline Mean-coExp & 2.13 & 1.66 \\
\hline Mean-tissues & 0.1 & 5.3 \\
\hline Description-tissues & "Immune system" & "Urine" \\
\hline Mean-BP & 0.1 & - \\
\hline Description-BP & $\begin{array}{l}\text { "regulation of inflammatory response" } \\
\text { "regulation of protein processing" } \\
\text { "regulation of protein maturation" } \\
\text { "regulation of protein binding" } \\
\text { "positive regulation of proteolysis" } \\
\text { "positive regulation of protein processing" }\end{array}$ & - \\
\hline Mean-TFs & 0.77 & - \\
\hline Description-TFs & $\begin{array}{l}\text { "Pax6", "PBX1", "Evi1", "NR3C1", } \\
\text { "Sox17", "NFATC2", "MIZF", "EBF1" }\end{array}$ & - \\
\hline Mean-GWAS & & 0.0022 \\
\hline Description-GWAS & "Acute kidney injury" & $\begin{array}{l}\text { "glomerular filtration rate" } \\
\text { "hypertension" } \\
\text { "urinary albumin to creatinine } \\
\text { ratio" } \\
\text { "serum albumin measurement" } \\
\text { "Microalbuminuria" }\end{array}$ \\
\hline prioritization score & 16.53 & 4.42 \\
\hline
\end{tabular}


Table 4 Table 3 (continued)

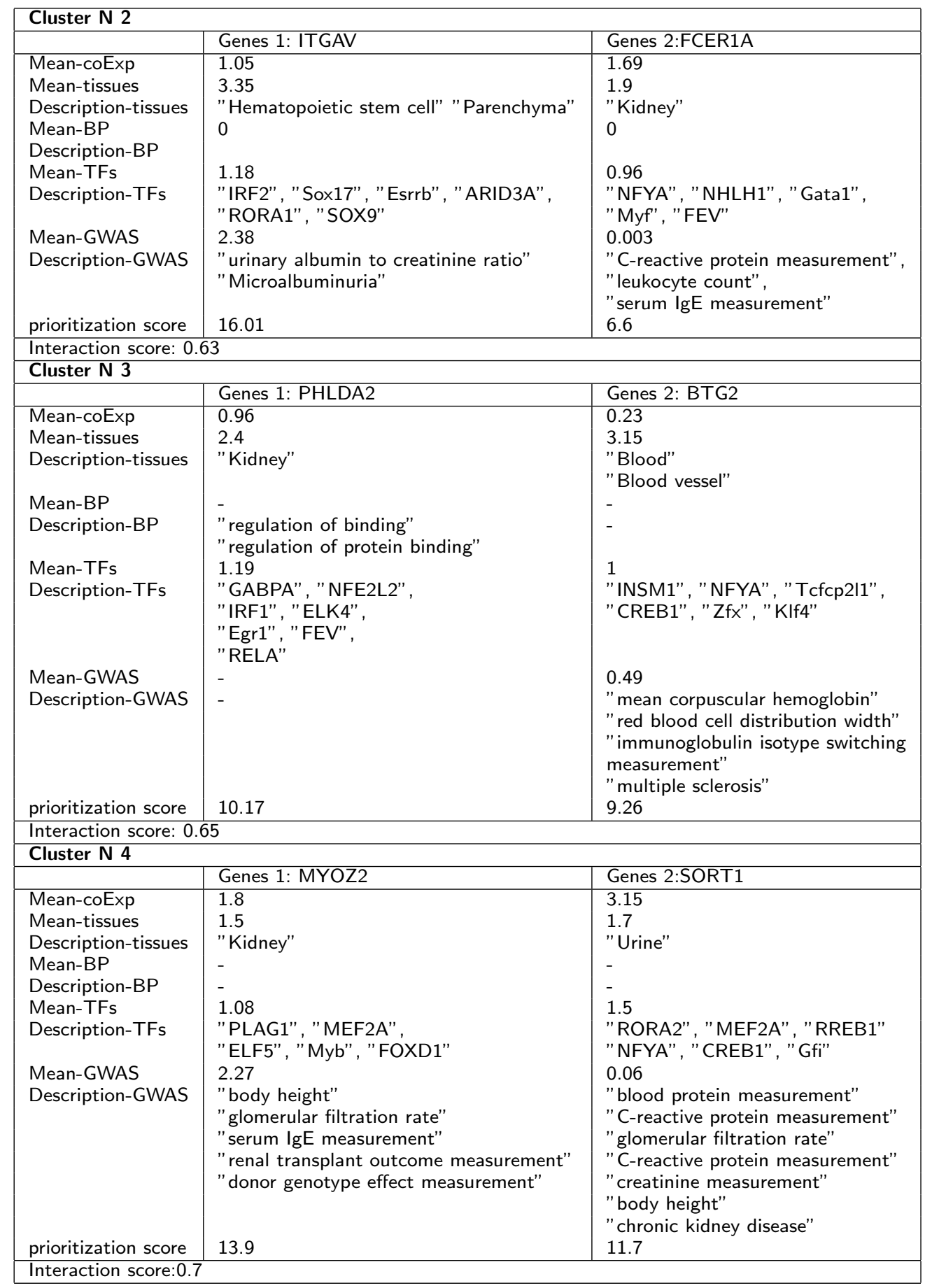

